\title{
DRL acquisition in rats with septal lesions'
}

MARJORIE CAPLAN AND JOHN STAMM

QUEENS COLLEGE OF THE CITY UNIVERSITY OF NEW YORK

Two groups of rats received DRL training according to one of two specified procedures. Each group included rats who were given bilateral lesions in the septal forebrain prior to the beginning of DRL training. It was found that acquisition of DRL timing behavior in animals with septal lesions is dependent upon the training method employed, or the specific delay interval required for reinforcement.

Several experiments have been conducted to determine the effects of septal lesions upon bar-pressing behavior of rats responding on a schedule for positive reinforcement. Schwartzbaum et al (1964) demonstrated that septal lesions markedly increased response rates in a discrimination task in the presence of the negative stimulus and during extinction, while response rates during the positive stimulus decreased slightly. Ellen \& Powell (1962) found that septal rats, when responding on a multiple schedule, exhibited, on the fixed interval component, a transitory loss in temporal discrimination and a permanent increase in terminal rates prior to the delivery of reinforcement. Response rates on the fixed ratio component did not change.

In a more recent study employing a schedule in which an animal is reinforced only for a response emitted after a predetermined delay interval (DRL), Ellen, Wilson, \& Powell (1964) found that acquisition of the required temporal discrimination was impaired in animals with septal lesions, but was comparable to normal in rats with callosal-cingulate, isocortical, or hippocampal lesions. These findings led the authors to conclude that septal lesions result in a decrement in the animal's ability to withhold a response.

The present study was conducted in an attempt to determine the conditions which result in a deficit in the temporal discrimination required on the DRL schedule.

\section{Method}

Nine male albino rats, approximately 10 months old at the beginning of the experiment, were reduced to $85 \%$ of their ad lib body weight and tested in a standard lever-pressing situation for food reinforcement (.045 gm Noyes pellets). Recording and programming of reinforcement contingencies were accomplished using BRS solid state modules. Sixteen counters were used to record time between successive responses, and interresponse time bin size was set at .1 the DRL value.

There were five dally sessions per week, at first $60 \mathrm{~min}$. per day and then $50 \mathrm{~min}$. per day. A water bottle was present in the experimental cage at all times during the session.

Ss were divided into two groups, with each group recelving DRL training according to a specified pro- cedure. Ss in the "gradual method" group $(\mathrm{N}=4)$ were shaped to press a bar in a Skinner box and then given $45 \mathrm{~min}$. training on a continuous reinforcement schedule. Two of these Ss were then given bilateral anodal lesions in the septal forebrain with $2 \mathrm{~mA}$ dc current passed for $30 \mathrm{sec}$. through a monopolar electrode, insulated except for .1 $\mathrm{mm}$ at the tip. The lesion was placed according to DeGroot coordinates $1.9 \mathrm{~mm}$ in front of Bregma, .6 mm lateral to the sagittal suture, and $4.8 \mathrm{~mm}$ below the surface of the skull at this point.

Approximately one week following surgery, these Ss were placed on a DRL 4 sec. schedule until they reached the criterion of $50 \%$ reinforced responses in one session. The interval was then lengthened in 2-sec. steps each time the animal reached a reinforcementto-response ratio of at least $50 \%$ at each successive interval.

Of the five Ss in the "abrupt method" group, three Ss were given lesions in the septal region prior to the beginning of the experiment. The five Ss were then shaped to press the bar in the Skinner box and immediately placed on a DRL 6 sec. schedule of reinforcement for one session, DRL $20 \mathrm{sec}$. for 10 sessions, then DRL $14 \mathrm{sec}$. for 10 sessions. The two normal animals only received training on DRL $6 \mathrm{sec}$. and DRL 20 sec.

\section{Results and Discussion}

Histological analysis of the brains showed complete destruction of the medial and lateral septal nuclei in all animals. The lesions extended to the medial parolfactory area, portions of the diagonal band of Broca, the bed nucleus of the anterior commissure, precommissural fibers of the fornix, and the septal accumbens nucleus.

The results for training by the "gradual" and the "abrupt" method are presented in Table 1 and Fig. 1. At the end of 50 sessions, one septal rat (MAC 19) was responding on a DRL schedule of $20 \mathrm{sec}$. and

Table 1. Mean reinforcement-to-response ratio for 4 different DRL values for two normal animals and two septal animals shaped by gradually increasing DRL value. Last day at each value.

(Ratio computed by dividing number of reinforcements obtained by number of responses made in one session).

\begin{tabular}{ccc} 
Interval Length (sec.) & Septals & Normals \\
\hline 6 & .78 & .71 \\
10 & .65 & .77 \\
14 & .65 & .75 \\
20 & $.55^{*}$ & .60 \\
\hline
\end{tabular}

* Only one animal represented here. 


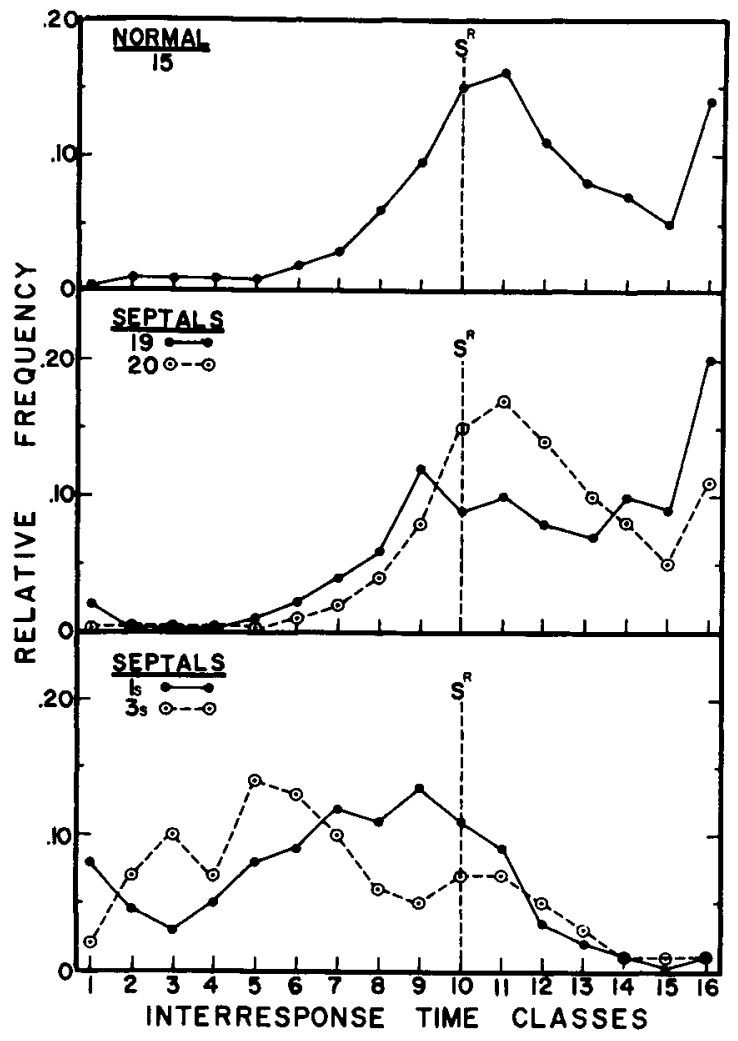

Fig. 1. ( $A$ and $B$ ). Relative frequency of interresponse times for two septal animals as compared to those of one normal after comparable running times on DRL 14 seconds; averaged over the last 4 days. (C). IRT distributions for two septal animals for whom the "abrupt" training procedure was used, following the same amount of training. (In all cases, bin size is equal to 1 the DRL value).

$55 \%$ of his responses were being rewarded, while the other operated S (MAC 20) had reached a contingency of $14 \mathrm{sec}$. with a reinforcement ratio of $56 \%$.

From these results, it can be seen that, with regard to acquisition, both the distribution of interresponse times and the percentage of reinforcements obtained by animals given septal lesions prior to introduction to the DRL schedule, are comparable to those of normal rats, provided acquisition proceeds gradually and the DRL contingency is lengthened by small discrete steps as the animal improves his timing behavior at each preceding one. If septal animals are required to meet a long delay contingency without this prior training, their distribution of IRTs demonstrates poor timing behavior and the relative number of reinforcements obtained is quite low.

It was also found that the relative number of reinforcements obtained by the septal animals trained according to the "abrupt" method was similar to that of the normal rats trained by the same method only if the DRL requirement was low, e.g., $6 \mathrm{sec}$. On this contingency, $64 \%$ of the septal Ss' responses were reinforced and $60 \%$ of the normal Ss' responses were reinforced. After the contingency was lengthened to 20 sec., on the last day of training, the relative number of reinforcements obtained was $59 \%$ for the normal rats, but only $9 \%$ for the septal rats.

These results indicate that the effects of septal lesions upon DRL timing behavior are dependent upon the training method employed, and the specific delay interval required for reinforcement, or both. Animals with lesions in the septal forebrain are able to suppress their responding to meet the DRL contingency only (1) if acquisition proceeds gradually, or (2) if the DRL requirement is low. This conclusion raises a question about the previous explanation given for septal animal's impairment, namely, as a "deficit in the ability to withhold a response" (McCleary, 1960; Schwartzbaum et al, 1964; Ellen, Wilson, \& Powell, 1964) and emphasizes the need to further specify the conditions existing within the situation which result in the particular effect observed.

\section{References}

Ellen, P., \& Powell, E. W. Effects of septal lesions on behavior generated by positive reinforcement. Exp. Neurol., 1962, 6, 1-11.

Ellen, P., Wilson, A. S., \& Powell, E. W. Septal inhibition and timing behavior in the rat. Exp. Neurol., 1964, 10, 120-132. McCleary, R. A. Response specificity in the behavioral effects of limbic system lesions in the cat. J. comp. physiol. Psychol., $1961,54,605-613$.

Schwartzbaum, J. S., Kellicutt, M. H., Spieth, T. M., \& Thompson. J. B. Effects of septal lesions in rats on response inhibition associated with food reinforced behavior. J. comp. physiol. Psychol., 1964, 58, 217-224.

\section{Note}

1. This research was supported in part by a grant given to Dr. John S. Stamm by the City University of New York. 